Tropical Journal of Pharmaceutical Research August 2020; 19 (8): 1701-1708

ISSN: $1596-5996$ (print); 1596-9827 (electronic)

(C) Pharmacotherapy Group, Faculty of Pharmacy, University of Benin, Benin City, 300001 Nigeria

\title{
Studies on the therapeutic effects of three TCM methods on steroid-induced osteonecrosis of femoral head
}

\author{
Xuyi Tan ${ }^{1,2}$, Xiantao Chen $^{3}$, Xiaobo Duan ${ }^{3}$, Shutu Gao ${ }^{3}$, Yuxin Guo ${ }^{1}$, Lu Huang ${ }^{4}$, \\ Gaoyan Kuang ${ }^{4}$, Min Lu ${ }^{4 *}$ \\ ${ }^{1}$ Hunan Academy of Chinese Medical Science, Changsha, Hunan 410006; ${ }^{2}$ Hunan University of Traditional Chinese Medicine, \\ Changsha, Hunan 410208; ${ }^{3}$ Luoyang Orthopedics Hospital of Henan Province, Luoyang, Henan 471000; ${ }^{4}$ Affiliated First \\ Hospital of Hunan University of Traditional Chinese Medicine, Changsha, Hunan 410007, China
}

*For correspondence: Email: lumin_62@163.com; Tel: +86-073185600441

Sent for review: 13 February 2020

Revised accepted: 19 July 2020

\begin{abstract}
Purpose: To determine the therapeutic effects of kidney yang-tonifying, blood-activating and phlegmeliminating methods on steroid-induced osteonecrosis of femoral head (SONFH), and their mechanisms of action.

Methods: A rabbit model of SONFH was established. Six rabbit groups were used: control, SONFH (given normal saline); tongluo shenggu group, kidney-tonifying group, blood-activating group, and phlegm--eliminating group, which received tongluo shenggu capsule $(0.28 \mathrm{~g} / \mathrm{kg} /$ day), yougui yin (YGY, $2.52 \mathrm{~g} / \mathrm{kg} /$ day), taohong siwu decoction (TSD, $2.38 \mathrm{~g} / \mathrm{kg} /$ day) and linggui zhugan decoction (LZD, 1.54 $\mathrm{g} / \mathrm{kg} /$ day), respectively, for 8 weeks. The following serum indices were assayed on the $2 \mathrm{nd}$, 4th and 8th weeks: total cholesterol, triglycerides, thromboxane B2 (TXB2), 6-keto-PGF1a, osteoprotegerin (OPG), and receptor activator of nuclear factor kappa-B ligand (RANKL). At the end of the 8th week, mRNA levels of OPG, BMP-2 and VEGF were assayed using real-time fluorescent quantitative polymerase chain reaction (RTF-qPCR).

Results: Serum OPG in the kidney-tonifying group was increased, while its RANKL was decreased on the 2nd week, but these parameters, along with $m R N A$ of OPG and BMP-2, reached normal levels at the 8 th week $(p<0.05)$. The TXB2 of blood-activating group was decreased, while its 6-keto-PGF1 level was increased at the 2nd week, but both of them attained normal levels at the 8th week, along with VEGF mRNA. Serum total cholesterol, triglycerides and TXB2 in the phlegm-eliminating group were decreased, while its 6-keto-PGF1a was increased at the 2 nd week, but the levels of serum total cholesterol and triglycerides were normalized at the 8th week $(p<0.05)$. Histological examination showed increases in bone trabeculae and bone marrow cells, while the proliferation of fat cells was decreased in kidney-tonifying, blood-activating and phlegm-eliminating groups.

Conclusion: These findings suggest that the therapeutic effects of the three different TCM methods on SONFH involve different mechanisms.
\end{abstract}

Keywords: Steroid-induced osteonecrosis, Femoral head, Yougui yin, Taohong siwu, Linggui zhugan decoction, Kidney-tonifying yang, Phlegm

\footnotetext{
This is an Open Access article that uses a fund-ing model which does not charge readers or their institutions for access and distributed under the terms of the Creative Commons Attribution License (http://creativecommons.org/licenses/by/4.0) and the Budapest Open Access Initiative (http://www.budapestopenaccessinitiative.org/read), which permit unrestricted use, distribution, and reproduction in any medium, provided the original work is properly credited.

Tropical Journal of Pharmaceutical Research is indexed by Science Citation Index (SciSearch), Scopus, International Pharmaceutical Abstract, Chemical Abstracts, Embase, Index Copernicus, EBSCO, African Index Medicus, JournalSeek, Journal Citation Reports/Science Edition, Directory of Open Access Journals (DOAJ), African Journal Online, Bioline International, Open-J-Gate and Pharmacy Abstracts
} 


\section{INTRODUCTION}

Steroid-induced osteonecrosis of femoral head (SONFH) refers to ischemic necrosis of femoral head caused by glucocorticoid application, resulting in hip joint pain and joint dysfunction. The disease is difficult to treat due to its insidious onset, and it is associated with many complications such as collapse of femoral head and osteoarthritis [1,2]. Thus, many patients undergo joint replacement and ultimately face the possibility of multiple revision surgeries.

The mechanism involved in the pathogenesis of SONFH is not well understood. However, it is generally believed to be related to three factors: bone metabolism, microcirculation disorders, and disorders in fat metabolism [3]. Previous studies have suggested that three of the most common TCM therapeutic methods i.e. kidney yangtonifying, blood-activating, and phlegmeliminating methods have good curative effects on SONFH [4].

However, the mechanisms involved in their therapeutic effects on SONFH, and their similarities or differences, have not been elucidated. The present study selected YGY, TSD and LZD to represent the three TCM therapeutic methods i.e. kidney yang-tonifying, blood-activating, and phlegm-eliminating methods, respectively. Their therapeutic effects on SONFH were investigated in a rabbit model of the disease, and their mechanisms of action were compared so as to provide experimental basis and theoretical support for their application in treatment of SONFH.

\section{EXPERIMENTAL}

\section{Animals}

A total of 114 healthy, big-eared adult New Zealand White rabbits weighing $2.0-2.5 \mathrm{~kg}$ were purchased from the Animal Laboratory of Luoyang Orthopedic Research Institute of Henan Province [production license $=$ SCXK (Jing) 2010-0001]. All animal studies (including euthanasia procedure) were done in line with Principles of Laboratory Animal Care [5], under the supervision of Luoyang Orthopedic Research Institute of Henan Province.

\section{Drugs}

In this study, YGY, which is used in TCM to warm kidney-nourishing yang, was used to represent kidney yang-tonifying method. It comprised the following Chinese herbs: shudihuang (cooked rehmannia, $15 \mathrm{~g}$ ); chaoshanyao (Chinese yam, $6 \mathrm{~g}$ ); shanzhuyu (Cornus officinalis, 3g); gouqi (wolfberry, $6 \mathrm{~g}$ ); duzhong (Eucommia ulmoides, $9 \mathrm{~g}$ ); rougui (cinnamon, $6 \mathrm{~g}$ ); zhifuzi (processed radix Aconiti lateralis, $6 \mathrm{~g}$ ); and zhi gancao (honey-fried licorice root, $3 \mathrm{~g}$ ). The blood-nourishing and blood-activating TSD comprised the following Chinese herbs: shudihuang (cooked rehmannia, $12 \mathrm{~g}$ ); danggui (Chinese angelica, $9 \mathrm{~g}$ ); baishao (white peony, $9 \mathrm{~g}$ ); chuanxiong (Ligusticum wallichii, $6 \mathrm{~g}$ ); taoren (peach kernel, $9 \mathrm{~g}$ ), and honghua (safflower, $6 \mathrm{~g}$ ).

Phlegm-eliminating method was represented with LZD which comprised the following Chinese herbs: fuling (Poria cocos, $12 \mathrm{~g}$ ); guizhi (cassia twig, $9 \mathrm{~g}$ ); baizhu (atractylodes, $6 \mathrm{~g}$ ), and zhigancao (honey-fried licorice root, $6 \mathrm{~g}$ ). These prescriptions were confirmed in Formulas of Chinese Medicine [6], and all the drugs were purchased in one batch from the TCM Pharmacy of Luoyang Orthopedic Hospital of Henan Province. Tongluo shenggu capsule was obtained from Zhejiang Haizheng Pharmaceutical Co. Ltd., catalog number: 20141009. The drug is usually used in TCM for invigoration of blood and bones, removal of blood stasis and relief of pain.

\section{Main reagents used}

The major reagents used, and their suppliers (in parenthesis) were: Trizol total RNA extraction reagent (Beijing Kangwei Century Biotechnology Co. Ltd, batch number: 2214L); Reverse transcription kits (Beijing Kangwei Century Biotechnology Co. Ltd, batch number: 00121411); UltraSYBR one-step fluorescence quantitative PCR kits (Beijing Kangwei Century Biotechnology Co. Ltd, batch number: $2913 \mathrm{~g}$ ), and rabbit Enzyme-linked immunosorbent assay (ELISA) kits for thromboxane B2, 6-keto-PGF1, OPG and RANKL (R\&D Company, USA, with the batch numbers: E1589, E1581, E1069 and E1342).

\section{Preparation of drug decoctions}

The drugs YGY, TSD and LZD were immersed in $500 \mathrm{~mL}$ of water for $30 \mathrm{~min}$, and then boiled with extra $500 \mathrm{~mL}$ water for $30 \mathrm{~min}$. Each decoction was kept. Then, the drugs were decocted once again following the same steps, and the two decoctions for each drug were combined and concentrated. Then, the decoctions were diluted to obtain crude drug concentrations of 0.252 , 0.238 and $0.154 \mathrm{~g} / \mathrm{mL}$ respectively. Tongluo shenggu capsule was dissolved in warm water to obtain a concentration of $0.028 \mathrm{~g} / \mathrm{mL}$. 


\section{Animal grouping}

A total of 114 New Zealand big-eared white rabbits were assigned to normal control group (comprising 19 rabbits) and model group (comprising 95 rabbits).

\section{Model preparation and verification}

The rabbit SONFH model was established according to the improved Matsui method [7]. The steps used were as follows: horse serum was injected at a dose of $10 \mathrm{~mL} / \mathrm{kg}$ via the ear marginal vein. Two weeks later, the injection was repeated at a lower dose $(5 \mathrm{~mL} / \mathrm{kg})$. Thereafter, 13 rabbits in the model group showed erythema at the ear margin, out of which 2 rabbits got anaphylaxis and died. Two weeks after the second injection of horse serum, the rabbits in the model group were given intraperitoneal injection of methylprednisolone succinate once a day at a dose of $40 \mathrm{mg} / \mathrm{kg}$ continuously for 3 days at 24-h intervals. To avoid infection, penicillin was injected along with methylprednisolone succinate at a dose of $100000 \mathrm{U} /$ piece, once a day.

Rabbits in normal control group received normal saline injection with the same method. Two weeks after the last injection of methylprednisolone succinate, one normal group rabbit and three model rabbits were selected randomly for verification of SONFH via magnetic resonance imaging (MRI) and hematoxylin-eosin $(\mathrm{H} \& \mathrm{E})$ staining of the femoral head.

\section{Drug intervention}

When the rabbit SONFH model was confirmed, there were 18 rabbits left in the normal control group. The remaining 90 rabbits in the model group were randomly divided into five groups (18 rabbits/group): model control group, tongluo shenggu group, kidney-tonifying group, bloodactivating group, and phlegm-eliminating group. The doses used were derived through conversion, with reference to the equivalent doses for humans [8]. Rabbits in the tongluo shenggu, kidney-tonifying, blood-activating and phlegm-eliminating groups were intragastrically administered tongluo shenggu capsule at a dose of $0.28 \mathrm{~g} / \mathrm{kg} /$ day, YGY $(2.52 \mathrm{~g} / \mathrm{kg} /$ day $)$, TSD (2.38 $\mathrm{g} / \mathrm{kg} / \mathrm{day}$ ) and LZD (1.54 g/kg/day), respectively. The rest were intragastrically administered equivalent volumes of physiological saline in place of drug. Six rabbits from each group were randomly selected for determination of biochemical indices and histological examination at weeks 2, 4 and 8 .

\section{Histological examination}

Histological examination of femoral head was carried out using a light microscope after H\&E staining.

\section{Total cholesterol and triglycerides}

Venous blood was taken from the ear vein and serum was obtained following centrifugation. Total cholesterol and triglycerides were determined using an automatic biochemical analyzer.

\section{Serum TXB2, 6-keto-PGF1a, OPG and RANKL}

Serum levels of TXB2, 6-keto-PGF1a, OPG and RANKL were assayed with ELISA in line with the manufacturer's protocols.

\section{Determination of OPG, BMP-2 and VEGF mRNA expression levels}

The mRNA expressions of OPG, BMP-2 and VEGF in femoral head necrosis tissue at the $8^{\text {th }}$ week were determined using RTF-qPCR. Trizol reagent was used for total RNA extraction from approximately $0.02 \mathrm{~g}$ of cryopreserved femoral head necrosis tissue, after which RTF-qPCR was performed following completion of RNA reverse transcription. The amplification conditions were 40 cycles of $95^{\circ} \mathrm{C}$ for $10 \mathrm{~min}, 95{ }^{\circ} \mathrm{C}$ for $10 \mathrm{sec}$, and $59^{\circ} \mathrm{C}$ for $50 \mathrm{sec}$. Melting curve acquisition range was from 60 to $95^{\circ} \mathrm{C}$. The mRNA expression level of each target gene was calculated with the $2^{-\Delta \Delta \mathrm{Ct}}$ method. The primers used for RT-qPCR amplification were provided by Nanjing Jinsirui Biotechnology Co. Ltd, and their sequences are shown in Table 1.

\section{Statistical analysis}

All experimental results were input into SPSS (version 16.0). Measurement data are presented as mean \pm standard deviation (SD). Measurement data that met the requirements of normality and homogeneity of variance were analyzed with one-way analysis of variance (LSD method). Values of $p<0.05$ were considered statistically significant.

\section{RESULTS}

\section{Histological observation}

As shown in Figure 1, bone trabeculae became thinner, bone marrow cells were decreased, and proliferation of fat cells was obvious in the model control group. Moreover, there were increases in bone trabeculae and bone marrow cells, while 
proliferation of fat cells decreased in the kidneytonifying group, blood-activating group, and phlegm-eliminating group at weeks 2, 4 and 8 .

Table 1: Sequence of primers used for RT-qPCR

\begin{tabular}{lll}
\hline $\begin{array}{l}\text { Target } \\
\text { gene }\end{array}$ & Primer & Length \\
\hline VEGF & Forward:5'- & \\
& $\begin{array}{l}\text { TCTTTCGGCGTGTTGCTCTG -3' } \\
\text { Reverse:5'- }\end{array}$ & \\
& 142bp \\
& GGTACATTTCGGGCGTGGTAT- & \\
OPG & Forward: 5'- & \\
& TTGCACCACTCCAAATCCAG-3' & \\
& 105bp \\
& Reverse: 5'- & \\
BMP & FATCGCACCCACAACCG-3' & \\
& Forward:5'- & \\
& GGCTGACCTGAGTGCCTGCGA & \\
& TA-3' & \\
& Reverse: 5'- \\
& GTCCTGAGCGAGTTCGAGTTG & \\
& CG-3' & \\
Actin & Forward: 5'- & \\
& CATCCTGCGTCTGGACCTGG-3' & \\
& Reverse: 5'- & \\
& TAATGTCACGCACGATTTCC-3' & \\
\hline
\end{tabular}

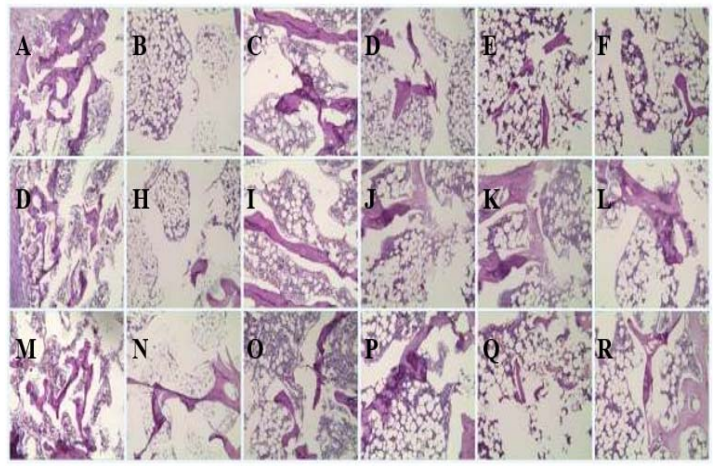

Figure 1: Photomicrographs of changes in each group after intervention (H\&E staining, $\times 100)$. A, D, and M: normal control group at $2^{\text {nd }} 4^{\text {th }}$ and $8^{\text {th }}$ weeks, respectively, showing absence of bone ischemic necrosis. $\mathbf{B}, \mathbf{H}$ and $\mathbf{N}$ : Model control group at weeks 2, 4 and 8 , respectively, showing thinner bone trabeculae, decreased bone marrow cell population, and proliferation of fat cells. C, I and $\mathbf{0}$ : Tongluo shenggu group at $2^{\text {nd }}, 4^{\text {th }}$, and $8^{\text {th }}$ weeks, respectively. $\mathbf{D}, \mathbf{J}$ and $\mathbf{P}$ : kidney-tonifying group at $2^{\text {nd }}, 4^{\text {th }}$ and $8^{\text {th }}$ weeks, respectively. $\mathbf{E}, \mathbf{K}$ and $\mathbf{Q}$ : blood-activating group at $2^{\text {nd }}, 4^{\text {th }}$ and $8^{\text {th }}$ weeks respectively. $\mathbf{F}, \mathbf{L}$ and R: phlegm-eliminating group at $2^{\text {nd }}, 4^{\text {th }}$ and $8^{\text {th }}$ weeks, respectively, showing increased bone trabeculae and bone marrow cells, while proliferation of fat cells was decreased in kidney tonifying group, blood-activating group, and phlegm-eliminating group at weeks 2, 4 and 8

\section{Levels of serum total cholesterol and triglycerides}

Relative to model control group, serum total cholesterol and triglycerides in phlegmeliminating group were decreased at $2^{\text {nd }}$ week ( $p$ $<0.05$ ), but they reached normal levels at the $4^{\text {th }}$ week $(p<0.05)$. Compared with the model control group, serum total cholesterol and triglycerides in tongluo shenggu group, kidneytonifying group, and blood-activating group were not significantly decreased at week 2 , but they were significantly decreased at week $4(p<$ 0.05 ). Serum total cholesterol and triglycerides in SONFH rabbits were higher than those in normal control rabbits. Treatment with YGY, TSD and LZD resulted in rapid and significant reductions in serum total cholesterol and triglycerides, as shown in Figure 2 and Figure 3.

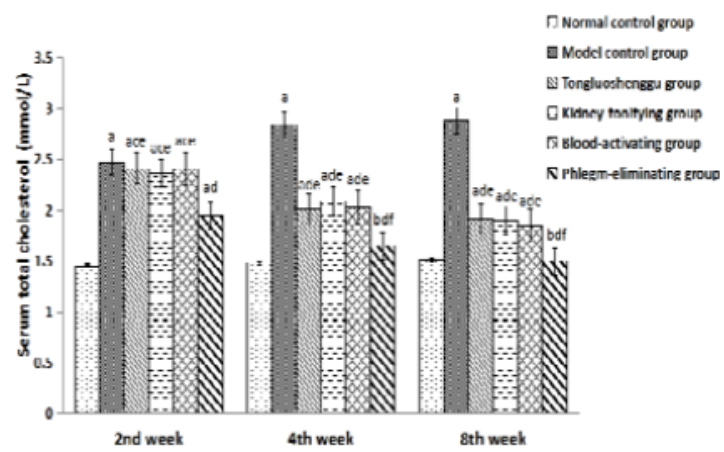

Figure 2: Serum total cholesterol of each group after intervention. $P>0.05$ : a, b , vs normal control

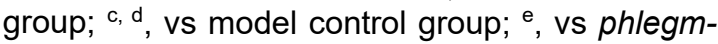
eliminating group; tongluo shenggu group, kidney-tonifying group, and blood-activating group compared with each other; ${ }^{f} p<0.05$, vs the $2^{\text {nd }}$ week intra-group

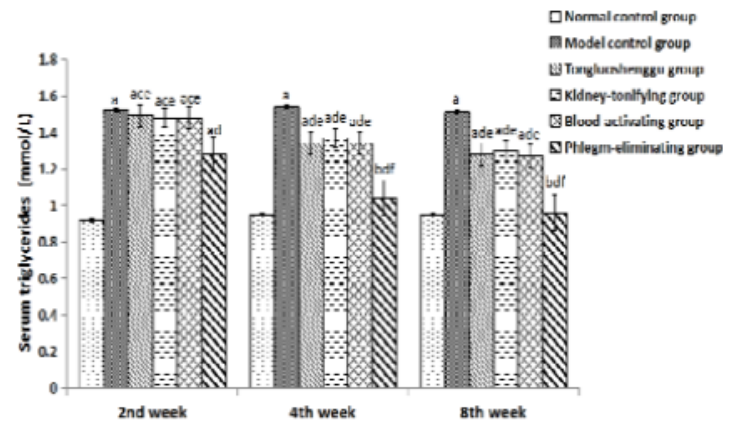

Figure 3: Serum triglycerides of each group after intervention. $P>0.05$ : a, b, vs normal control group; c, d, vs model control group; e, vs phlegm-eliminating group; tongluo shenggu group, kidney-tonifying group and blood-activating group compared with each other, $p>0.05 ;{ }^{\text {f }} P<0.05$, vs the $2^{\text {nd }}$ week intra-group

\section{Serum TXB2 and 6-keto-PGF1a}

Serum TXB2 level in blood-activating group was decreased and its 6-keto-PGF1a was increased at the $2^{\text {nd }}$ week, but these parameters attained

Trop J Pharm Res, August 2020; 19(8): 1704 
normal levels at the $8^{\text {th }}$ week. Compared to model control group, serum TXB2 and 6-ketoPGF1a in phlegm-eliminating group were significantly changed at the $2^{\text {nd }}, 4^{\text {th }}$ and $8^{\text {th }}$ weeks ( $p<0.05)$, but they did not return to normal levels. In the kidney-tonifying group, serum levels of TXB2 and 6-keto-PGF1 $\alpha$ were significantly changed at the $4^{\text {th }}$ and $8^{\text {th }}$ weeks $(p<0.05)$. Serum TXB2 in SONFH rabbits were higher than that in normal rabbits, while 6-keto-PGF1a was lower. However, YGY, LZD and TSD significantly and rapidly reduced serum $\mathrm{TXB} 2$, and increased 6-keto-PGF1a levels in SONFH rabbits, as shown in Figures 4 and 5.

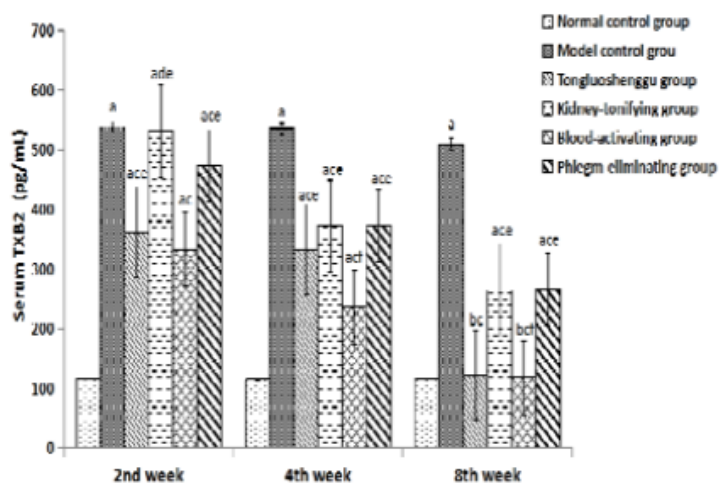

Figure 4: Serum TXB2 of each group after intervention. $P,>0.05$ : a, b, vs normal control group; ${ }^{c}$,

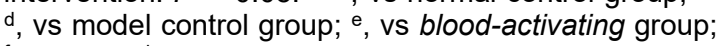
f, vs the $2^{\text {nd }}$ week intra-group

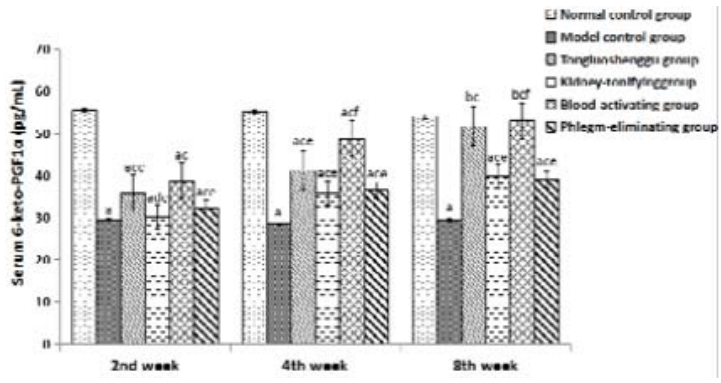

Figure 5: Serum 6-keto-PGF1 $\alpha$ level in each of the groups after intervention. $P>0.05$ : a, b, vs normal controlgroup; ${ }^{c}$, d, vs model control group; ${ }^{e}$, vs bloodactivating group; ${ }^{f}$, vs the $2^{\text {nd }}$ week intra-group

\section{Serum OPG and RANKL levels}

Serum OPG of the kidney-tonifying group was increased, while its RANKL was decreased at the $2^{\text {nd }}$ week $(p<0.05)$, but the values of these parameters reached normal levels at the $4^{\text {th }}$ week. Relative to model control group, values of serum OPG and RANKL in the blood-activating group and phlegm-eliminating group were significantly changed at $4^{\text {th }}$ and $8^{\text {th }}$ weeks $(p<$ $0.05)$, but they did not return to normal levels at the $8^{\text {th }}$ week. Serum RANKL level in the SONFH model rabbits was markedly higher than that in normal control rabbits, while OPG level was lower. However, treatment with TSD, LZD and YGY led to significant and rapid reduction in serum RANKL, and marked increase in OPG. These results are shown in Figures 6 and 7.

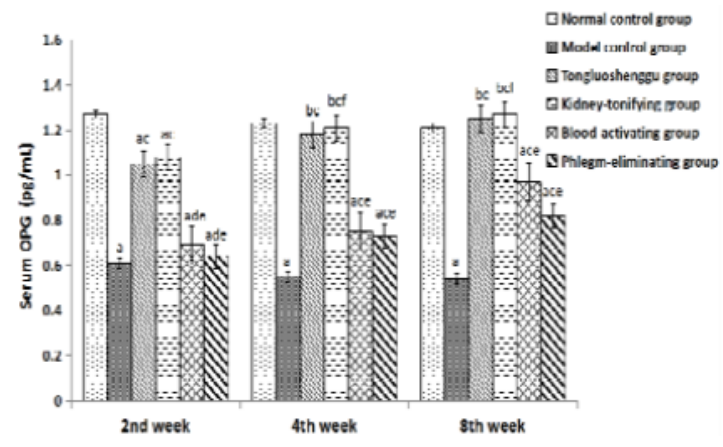

Figure 6: Serum OPG of each group after intervention. $P>0.05$ : a, b, vs normal control group; $\mathrm{c}, \mathrm{d}$, vs model control group; e, vs kidney-tonifying group; blood-activating group and phlegm-eliminating group compared with each other, $p>0.05 ;{ }^{f} p<0.05$, vs the $2^{\text {nd }}$ week intra-group.

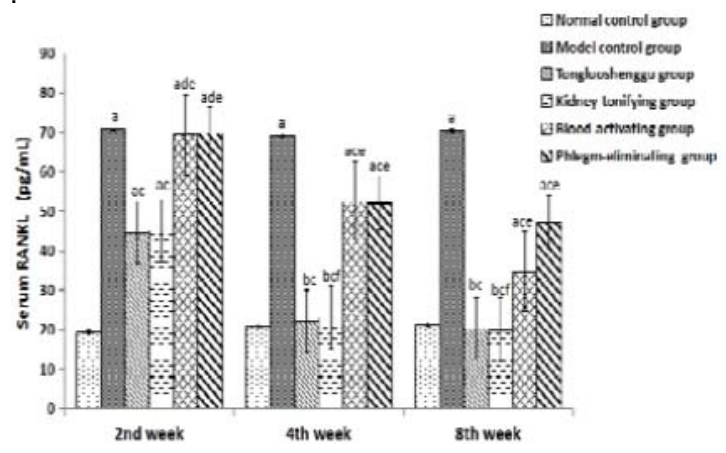

Figure 7: Serum RANKL of each group after intervention. $P>0.05$ : a, b, vs normal control group; c, d, vs model control group; e, vs kidney-tonifying group; blood-activating group and phlegm-eliminating group compared with each other, $p>0.05$; ${ }^{f} p<0.05$, vs the $2^{\text {nd }}$ week intra-group.

\section{Expression levels of mRNAs for OPG, BMP-2 and VEGF}

Relative to the model control group, levels of OPG, BMP-2 and VEGF mRNA in femoral head of kidney-tonifying, blood-activating and phlegmeliminating groups were significantly upregulated at the $8^{\text {th }}$ week $(p<0.05)$. Moreover, mRNA levels of OPG and BMP-2 in kidney-tonifying group and VEGF mRNA of blood-activating reached normal levels at the $8^{\text {th }}$ week. The data showed that the mRNA expression levels of OPG, BMP-2 and VEGF in the femoral head necrotic tissue of SONFH rabbits were lower than those of normal rabbits. However, YGY 
significantly upregulated mRNAs of OPG and BMP-2, while TSD significantly upregulated VEGF mRNA. These results are presented in Figure 8.

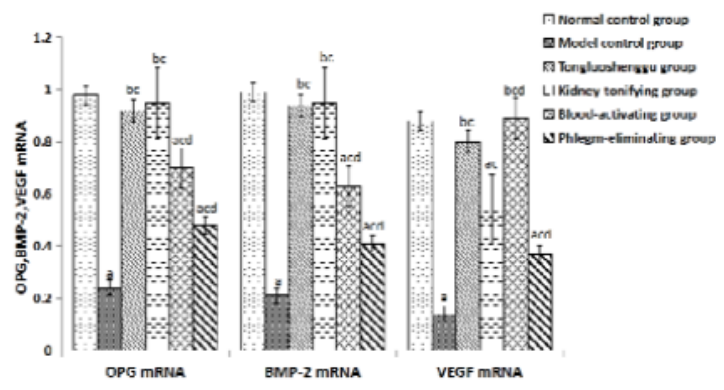

Figure 8: mRNA expression levels of OPG, BMP-2 and VEGF in each group at week $8 .{ }^{a, b} P>0.05$, vs normal control group; ${ }^{c} p<0.05$, vs model control group; ${ }^{d} p<0.05$, vs kidney-tonifying group

\section{DISCUSSION}

Steroid-induced osteonecrosis of femoral head (SONFH) refers to ischemic necrosis of femoral head caused by high dose of glucocorticoids. However, the exact mechanism underlying the pathogenesis of SONFH has not been fully elucidated. Currently, there are a few theories about the pathogenesis of SONFH. These theories relate to osteoporosis, apoptosis, intraosseous hypertension, intravascular coagulation, and disorders in lipid metabolism, all of which are closely associated with bone metabolism, microcirculation, and lipid metabolism [9, 10]. Therefore, the clinical treatment of SONFH is based on regulation of bone metabolism using bisphosphonates, regulation of lipid metabolism with statins, and increasing blood supply to the femoral head using anticoagulants and vasodilators [11].

There is no record of SONFH in TCM. However, according to characteristics of its pathogenesis, SONFH falls under the category of TCM diseases such as "Gu shi", "Gu wei" and "Gu bi". In the same way SONFH has a variety of TCM names, there are also different emphases when it comes to research on its pathogenesis in TCM. In TCM, the three-period and four-pattern syndrome differentiation standards proposed by Professor Chen Wei-heng are generally recognized [12]. At present, most scholars engaged in research on the pathogenesis of SONFH recognize the involvement of "virtual", "blood stasis" and "phlegm" in the process [13]. A review of the literature suggests that the three most common TCM therapeutic methods used against SONFH i.e. kidney yang-tonifying, bloodactivating, and phlegm-eliminating methods produce good curative effects.
It had been reported that different therapeutic methods produced different onset times and therapeutic effects in the treatment course of SONFH [14]. Some scholars reported that kidney-tonifying drugs inhibited the activation of osteoclasts in a rat model of SONFH, and increased bone formation by increasing serum osteocalcin and calcitonin levels [15]. Other scholars have found that blood-activating drugs enhance blood circulation, thereby removing blood stasis, improving femoral head microcirculation, and promoting repair of necrotic tissue. These findings highlight the significance of promoting circulation and removing stasis in the treatment of SONFH [16].

Some results have shown that the use of glucocorticoids by patients gave rise to disorders in fat metabolism through reduction in osteogenetic differentiation, and induction of changes in the metabolism of bone marrow mesenchymal stem cells in the direction of lipogenesis. This would lead to increases in blood lipid levels and disorders in microcirculation, resulting in blockage of tiny blood vessels. Therefore, the clinical treatment of SONFH should take into account the elimination of phlegm as "phlegm wet" in the etiology of SONFH. Thus, in this study, it was assumed that the three TCM therapeutic strategies i.e. kidney-tonifying, bloodactivating and phlegm-eliminating methods may have different effects on regulation of bone metabolism, lipid metabolism and blood supply to femoral head. Thus, their respective effects on SONFH may involve different mechanisms.

Studies have shown that YGY increased serum calcium and phosphorus levels, promoted proliferation of bone marrow mesenchymal stem cells, and enhanced osteogenesis differentiation in SONFH models, thereby increasing bone mineral density [17-20]. Thus, YGY has a very good therapeutic effect on SONHF. Taohong siwu decoction comes from yizong jinjian which is used in TCM to nourish the blood and promote blood circulation. Previous studies reported that taohong siwu decoction upregulated the transcription of transforming growth factor- $\beta 1$ (TGF- $\beta$ ) mRNA, enhanced the regeneration of local microvessels, and exerted therapeutic effect on SONFH [21,22].

The therapeutic effect of TSD prescription on SONFH may occur via rapid regulation of microcirculation indices and increased blood supply to the femoral head. In contrast, the therapeutic effect of LZD prescription on SONFH may involve rapid regulation of lipid metabolism and reductions in blood viscosity and fat embolus formation in blood vessels of femoral head. Thus, 
the three different TCM therapeutic methods may have different therapeutic mechanisms of action on SONFH. These are summarized in Figure 9.

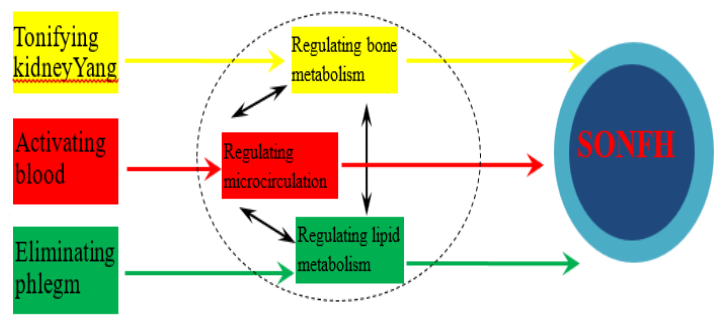

Figure 9: Schematic illustration of mechanisms of action of three different TCM therapeutic methods on SONFH

In the book Chinese Orthopaedics and Traumatology Science, LZD which comes from jingui yaolue, is recommended for use against SONFH when syndrome differentiation involves phlegm [23]. It has been reported that LZD effectively reduced serum levels of triglycerides, total cholesterol and high-density lipoprotein [24]. These findings explain the bases for the choice of YGY, TSD and LZD to represent kidney yangtonifying, blood-activating and phlegmeliminating methods, respectively, in this study.

\section{CONCLUSION}

The results obtained in this study suggest that YGY, TSD and LZD exert therapeutic effects on SONFH through different mechanisms. Based on the findings, kidney yang-tonifying YGY prescription may exert its effect on SONFH via rapid regulation of indicators of bone metabolism and promotion of the formation of bone tissue, resulting in the repair of necrotic areas.

\section{Limitations of the study}

There are some limitations in this study. This is essentially a preliminary study on the mechanisms involved in the therapeutic effects of three different TCM methods on SONFH. Only human equivalent doses were used in the study. Thus, it was not possible to obtain information on dose-effect relationships. In addition, the effects of the various prescriptions on signaling pathways were not studied. This makes it difficult to relate the mechanisms of action to signaling pathways. Therefore, in subsequent studies, dose screening will be used to determine the appropriate animal doses for use in identification of the associated signal pathways, with a view to achieving a clearer elucidation of the mechanisms underlying the therapeutic effects of the three TCM methods on SONFH.

\section{DECLARATIONS}

\section{Acknowledgement}

This study was supported by Henan TCM Scientific Research special key project (No. 2014ZY01008), the Postdoctoral Research Program of Hunan university of TCM (No. 194780), and State Administration of Traditional Chinese Medicine "National Famous Old Chinese Medicine Expert Qiu Xiang-Zhong Inheritance Studio Construction Project" [No.42(2016)]. The funds covered experimental costs and publication fee.

\section{Conflict of interest}

No conflict of interest is associated with this work.

\section{Contribution of authors}

We declare that this work was done by the authors named in this article and all liabilities pertaining to claims relating to the content of this article will be borne by the authors.

\section{Open Access}

This is an Open Access article that uses a funding model which does not charge readers or their institutions for access and distributed under the terms of the Creative Commons Attribution License (http://creativecommons.org/licenses/by/ 4.0) and the Budapest Open Access Initiative (http://www.budapestopenaccessinitiative.org/rea d), which permit unrestricted use, distribution, and reproduction in any medium, provided the original work is properly credited.

\section{REFERENCES}

1. Microprosthodontics group of orthopedics branch of Chinese medical association, Group of osteonecrosis and osteonecrosis, Chinese professional committee of reconstructive surgery. Standard expert consensus on the diagnosis and treatment of adult femoral head necrosis (2012 edition). Chin J Orthop 2012; 32(6): 606611.

2. Chen $X T$, Tan $X Y$, Gao ST, Zhang XD, Li JM, Liu YW. Sartorius muscle-pedicle bone graft for osteonecrosis of the femoral head. Int Orthop 2016; 40(7): 1417-1425

3. Moya-Angeler J, Gianakos AL, Villa JC, Ni A, Lane JM. Current concepts on osteonecrosis of the femoral head. World J Orthop 2015; 6(8): 590-601

4. Tan XY, Li G, Gao ST, Cheng ChS, Liu LY. Advances of Experimental Research on TCM for Steroid-induced 
Necrosis of Femoral Head. Guiding J Tradit Chin Med Pharm 2014; 20(12): 49-52

5. World Health Organization. Principles of laboratory animal care. WHO Chron 1985; 39: 51-56

6. Deng ZJ. Formulas of Chinese Medicine. Beijing: Chin Tradit Chin Med Press 2003; pp 162, 180, 309

7. Matsui $M$, Saito $S$, Ohzono $K$, Sugano $N$, Saito $M$, Takaoka K, Ono K. Experimental steroid-induced osteonecrosis in adult rabbits with hypersensitive vasculitis. Clin Orthop and Related Res 1992; 277 (4): 61-72.

8. Wei Wi, Wu XM, Li YJ. Pharmacological experimental methodology (4th edition). Beijing: People's Medical Publishing House 2010: 1698.

9. Gillet $C$, Dalla VA, Gaspard $N$, Spruyt $D$, Vertongen $P$, Lechanteur J, Rigutto S, Dragan ER, Heuschling A, Gangji V, Rasschaert J. Osteonecrosis of the Femoral Head: Lipotoxicity Exacerbation in MSC and Modifications of the Bone Marrow Fluid. Endocrinol 2017; 158(3): 490-502.

10. Houdek MT, Wyles CC, Packard BD, Terzic A, Behfar A, Sierra RJ. Decreased Osteogenic Activity of Mesenchymal Stem Cells in Patients With Corticosteroid-Induced Osteonecrosis of the Femoral Head. J Arthroplasty 2016; 31(4): 893-898

11. Zhang CQ. Guidelines for hip preservation treatment of femoral head necrosis (2016 edition). Chin J Geriatr Orthop and Rehabil (Electron Ed) 2016; 2(2): 65-70.

12. Chen WH, Liu DB, Zhang HM, Gu LJ, SunG, Zhao TJ, Zhang L, ZhangQ. Thought or Syndrome Differentiation in Necrosis of the Femoral Head by Third-periods and Fourth-Patterns. Chin J Basic Med Tradit Chin Med 2003; 9(12): 51-52

13. Wang XM, Cao LZ, Zhang XG, Zhang HI, Guo CHG, Qin $D P$, Su LL, Wu MJ. Research progress in TCM syndromes of steroid-induced avascular necrosis of the femoral head using proteomics, genomics, and metabolomics. Chin J Osteoporosis 2018; 24(7): 966970.

14. Wang RT, Lin SHF, Wan R, Yin XJ, Wang ZHY, Liu DB, Lin $N$, Chen $W H$. Effects of different therapeutic methods and prescriptions on blood fat, blood viscosity, blood coagulation and fibrinolysis of chickens with steroid-induced necrosis of femoral head. J Tradi Chin Orthop Traumatol 2013; 25(3): 21-30.

15. Wang $H$, Liu CHF, Jiang YN, Chen WH, Lin N. Bone Repair Effect of Bushen Formula on Rats with Steroidinduced Osteonecrosis of Femoral Head. Chin J Exp Tradi Med Formulae 2016; 22(1): 88-92.

16. He W, Xu CHY, Fan YG, Fang B, Li X, Wang HB, Liu $S H J$, Yuan $H$. Experimental study of the influence of blood-circulation-activating traditional Chinese drugs on plasma TXB2 and 6-keto-PGF1a of glucocorticoidinduced femoral head necrosis. China J Orthop Traumatol 2002; 15(9): 531-533.

17. Song CY, Shen $X C H$, Lu SHJ, Bao HX, Jin HT, Xiao LW, Tong PJ. Effect of YGY on the Blood Bone Mineral Salt and Bone Mineral Density in Rats with Femoral Head Necrosis. Journal of Tradit Chin Med 2015; 56(14): 1239-1242.

18. Shen XC, Song CY, Lyu SJ, Bao HX, Tong PJ, Xiao LW. Experimental study on preventive effect of Yougui drink on femoral head necrosis in rats under micro CT. China J Orthop Traumatol 2015; 28(12): 1106-10

19. Hu MF, Zhou XC, Shan LT, Xiao LW, Tong PJ. Effects of Yougui recipe on the behavioral changes in rat of steroid-induced avascular necrosis of the femoral head. China J Orthop Traumatol 2013; 26(1): 50-3

20. Wu YG, Xiao LW, Tong PJ. Effects of yougui drink on the osteogenic differentiation of mesenchymal stem cells in the proximal femur in steroid-induced avascular necrosis of femoral head. China J Orthop Traumatol 2009; 22(2): 115-7

21. Huang CHY, Sun SHQ, Li YL. Effects of TSD on Angiogenin of Rabbit Skin Graft Models. J Hunan Univ Chin Med 2015; 35(11): 6-9.

22. Chen LL, Chen Xb, Hong GJ, Chen D, Yang P, He W. "Removing Blood Stasis" Method for bone repair in steroid-induced osteonecrosis of the femoral heads. Chin J Tissue Eng Res 2016; 20(15): 2148-2155.

23. Wang HM. Chinese orthopaedics and traumatology science (2nd edition). BEIJING: Chin Tradit Chin Med press 2007: 339.

24. Yang Y, Qin J, Ke B, Zhang J, Shi L, Li Q. Effect of Linguizhugan decoction on hyperlipidemia rats with intermittent fasting. J Tradit Chin Med 2013; 33(2): 250252. 